\title{
Double tailgut cysts (presacral and precoccygeal hamartomas) with CA 19-9 elevation
}

\author{
Ji Won Kim ${ }^{1}$, Jae Woong Han ${ }^{1}$, So Young Jung ${ }^{1}$, Seung Chul Lee ${ }^{1}$, Byung Chun Kim ${ }^{1 *}$, \\ Hye Kyung Ahn ${ }^{2}$, Han Myun Kim ${ }^{3}$ \\ ${ }^{1}$ Departments of Surgery, Kangnam Sacred Heart Hospital, Hallym University College of Medicine, Seoul, South Korea; \\ *Corresponding Author: kimjiw96@naver.com \\ ${ }^{2}$ Departments of Pathology, Kangnam Sacred Heart Hospital, Hallym University College of Medicine, Seoul, South Korea \\ ${ }^{3}$ Departments of Radiology, Kangnam Sacred Heart Hospital, Hallym University College of Medicine, Seoul, South Korea
}

Received 2 December 2012; revised 1 February 2013; accepted 7 March 2013

\section{ABSTRACT}

Tailgut cyst, also called retrorectal hamartoma, is a rare congenital lesion and is usually located anterior to the sacrum and posterior to the rectum. We report a case of double tailgut cyst (presacral and precoccygeal hamartoma). A 62-yearold female visited the hospital complaining of anal pain and pressure in the perianal area for one month. Digital rectal examination revealed a round mass with hard consistency in the lateral and posterior part of rectum $5 \mathrm{~cm}$ from the anal verge. Pelvic magnetic resonance imaging showed two cystic and solid presacral masses (lateral and posterior part of rectum) compressing the rectum. The patient underwent surgical resection for pathologic diagnosis. At operation, two masses were located in the left presacral space without invasion to adjacent organs and soft tissue. Pathologic examination revealed double tailgut cysts without malignant transformation. A differential diagnosis of perirectal cystic hamartoma includes epidermal cysts, cystic teratomas, dermoid cysts, anal gland cysts, sarcoma and rectal duplications. Regional inflammatory process frequently complicates this lesion and perirectal fistula can develop. Tailgut cyst also has a malignancy potential, with development of adenocarcinomas. To establish a definite diagnosis and prevent complication, complete surgical resection is recommended.

Keywords: Tailgut Cyst; Retrorectal Hamartoma; Presacral Tumor; Retrorectal Tumor; Retrectal Developmental Cysts

\section{INTRODUCTION}

Tailgut cyst, also known as retrorectal hamartoma and mucus-secreting cyst, is a rare, congenital developmental disease that arises from the remnants of the embryonic postanal gut with incomplete regression during development. It almost invariably occurs within the retrorectal or presacral space, and rarely in the perirenal area, subcutaneous tissue in the anorectal area and prerectal region $[1,2]$. Tailgut cyst can be easily misdiagnosed because the symptoms are similar to those of inflammatory disease, such as pilonidal abscess, perianal abscess and complicated fistula. If the initial operation for a tailgut cyst is performed inappropriately, correct diagnosis and complete excision will be more difficult. Herein, we report the case of a double tailgut cyst and present a review of the relevant literature to emphasize the importance of consideration of double tailgut cyst as a differential diagnosis in evaluating patients complaining of symptoms suggestive of benign anal inflammatory disease.

\section{CASE REPORT}

A 62-year-old female patient presented with anal pain and pressure in the perianal area for one month. The past medical history revealed a thyroidectomy and hemorrhoidectomy. On digital rectal examination, an indurated mass with round shape was palpated. Routine laboratory tests including a complete blood cell count, electrolytes and routine urinalysis were within normal limits. Serum tumor markers including carcinoembryonic antigen (CEA), alpha-fetoprotein and lactic dehydrogenase were within normal range, but the tumor marker carbohydrate antigen (CA) 19-9 level was $102.2 \mathrm{U} / \mathrm{mL}$. Pelvic magnetic resonance imaging (MRI) with contrast demonstrated a $6.5 \times$ $4.5 \mathrm{~cm}$ presacral cystic and solid mass with hypointensity on T1-weighted images and hyperintensity T2-weighted images in the left posterolateral perirectal area, displacing the rectum and a $2.2 \times 2.1 \mathrm{~cm}$ cystic dominant mass in the precoccygeal space with hypointensity on T1weighted image and hyperintensity on T2-weighted images, closely abutting to the posterior rectal wall and ex- 
tending to the ischiorectal fossa beyond the pelvic floor (Figure 1). Positron emission tomography (PET) scan showed that the two masses located in the presacral and precoccygeal area were isometabolic (Figure 2). The patient underwent explorative laparotomy for the definite pathologic diagnosis and treatment. Intraoperatively, the two masses did not communicate with the bladder or rectum. The larger tumor extended from the presacral space to the left perirectal space and pushed the rectum to the right pelvic area. The smaller tumor was located in the precoccygeal space. The tumors were not densely adherent to the sacrum and coccyx, and were not adherent to the perirectal fat. Complete excision of the tumors was performed and there was no injury to the rectum. On histopathological examination, the larger presacral mass was revealed as a cystic mass, measuring $6.5 \times 5 \times 3.5$ $\mathrm{cm}$. The cut surface showed whitish irregular nodules, measuring up to $3 \times 3 \mathrm{~cm}$ and was filled with yellowish brown necrotic fluid. The cut surface of the smaller precoccygeal mass measured $2.7 \times 2.5 \times 0.9 \mathrm{~cm}$ and had a white, partially cystic and solid appearance (Figure 3). Microscopically, the masses were composed of multiloculated cystic spaces lined by pseudostratified columnar to cuboidal epithelium surrounded by smooth muscle wall. Squamous epithelium was focally present (Figure 4A). Some cystic spaces were lined by mucin secreting columnar epithelium with goblet cells (Figure 4B). The final diagnosis was tailgut cysts without malignant transformation. The patient was discharged 7 days after surgery without complications.

\section{DISCUSSION}

Retrorectal tumors are rare and are usually congenital

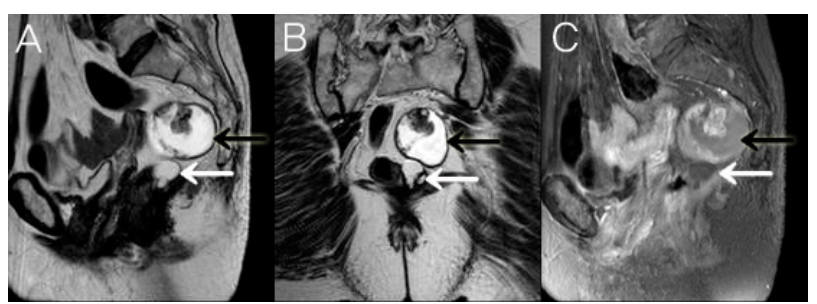

Figure 1. Sagittal T2 weighted (A), Axial T2 weighted (B) and Sagittal post-contrast $\mathrm{T} 1$ weighted fat saturation (C) magnetic resonance images revealing two lesions (larger cyst-black arrow, smaller cyst-white arrow) located in the retrorectal space. The rectum was displaced right laterally owing to a mass effect from a large main lesion. The diameter of the lesions was $6.5 \times$ $4.5 \mathrm{~cm}$ and $2.2 \times 2.1 \mathrm{~cm}$. Two masses were hypointense on T1-weighted MRI and hyperintense on T2-weighted MRI. These lesions were multicystic masses with a well defined margin and internal solid portions. Internal solid portion and thin regular peripheral rim were hypointense on all sequences. After gadolinium administration, the solid portions and the rim were moderate enhancement. There was no evidence of bony involvement or adjacent invasion.

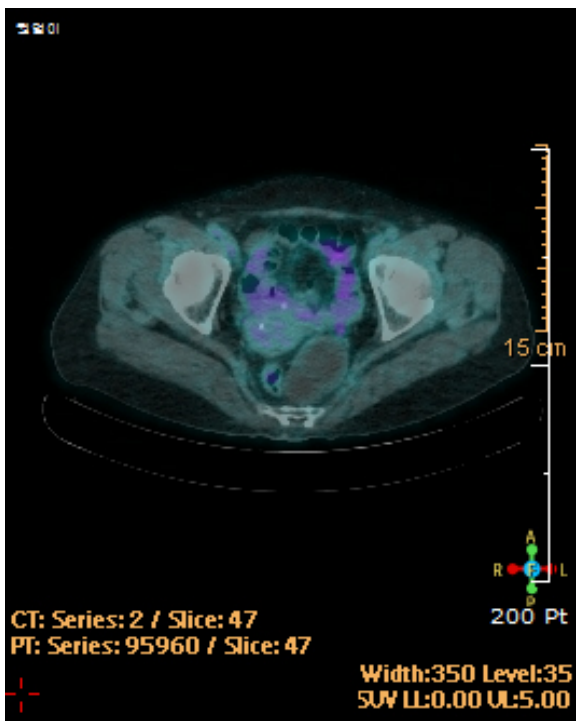

Figure 2. PET-CT demonstrated no FDG uptake of the masses.

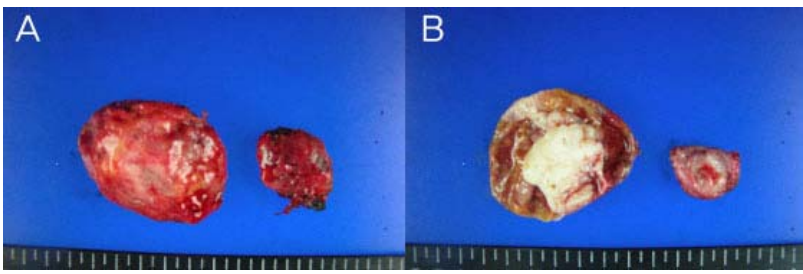

Figure 3. Gross appearance (A) and cut surface (B) of resected tailgut cysts.

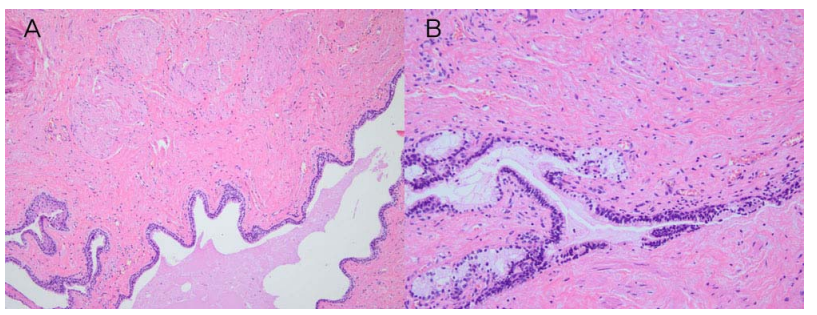

Figure 4. Microscopic pathologic findings. A (magnification $\times$ $100)$, B (magnification $\times 200)$.

developmental cysts [1-3]. Congenital developmental cysts, according to their origin and histologic features, include dermoid cysts, epidermoid cysts, enteric cysts and neurenteric cysts [4]. Tailgut cysts, which are also known as retrorectal cystic hamartomas, are rare congenital, multicystic, enteric lesions that lie between the rectum and the sacrum, and which are bounded superiorly by the peritoneal reflection, inferiorly by the levator ani and coccygeus muscles and laterally by the ureters and iliac vessels [5]. Most tailgut cysts are asymptomatic and are often discovered incidentally during physical examination. The compressive symptom due to the growing mass is present in $50 \%$ of patients and includes changes in stool caliber, rectal fullness, bleeding, constipation, 
lower abdominal and back pain and urinary frequency, and is frequently associated with infected tailgut cyst with rectal fistula or anal fistula [6]. Tailgut cysts can be initially misdiagnosed. When a patient comes to the hospital presenting with a procedural history of multiple drainage for recurrent pilonidal abscess, perianal abscess or anal fistula, tailgut cyst should be suspected [7]. Tailgut cyst can affect a person at any age but predominantly affects middle-aged women [1-6]. The differential diagnosis of tailgut cyst includes cystic sacrococcygeal teratoma, epidermal cyst, dermoid cyst, neuroenteric cyst, rectal duplication, anal gland cyst, neuroblastoma, necrotic rectal leiomyosarcoma, extraperitoneal adenomucinosis, anterior sacral meningocele, necrotic sacral chordoma, cystic lymphangioma, pyogenic abscess and adnexal tumor [8]. Preoperative diagnosis is very difficult and various diagnostic methods are used. Pelvic MRI is the imaging method of choice in differentiating tailgut cysts from other lesions [6,9]. Tailgut cysts usually have a low signal intensity on T1-weighted images and high signal intensity on T2-weighted images $[9,10]$. MRI is more sensitive than computed tomography (CT) scan in differentiating unilocular and multilocular tumors and especially for the detection of small peripheral cysts [10]. CT is also considered a good diagnostic method. Detailed physical examination including a digital rectal examination can enable the correct diagnosis before operation [11]. Transabdominal or transrectal ultrasound, especially in the case of tailgut cyst occurring in females, can led to a misdiagnosis as adnexal tumors such as dermoid cysts [4]. A diagnostic biopsy is not recommended due to the risk of spillage of tumor cells, spread of infection into the abdominal cavity and missed detection of malignant foci [12]. Tailgut cysts are usually benign and malignant transformation is rare. Cases of malignancies include adenocarcinoma, carcinoid tumors, neuroendocrine carcinoma, endometrioid carcinoma, adenosquamous carcinoma, squamous cell carcinoma and sarcoma have been reported to be associated with tailgut cyst and adenocarcinoma, and carcinoid tumors are the most common malignant transformation pattern of tailgut cysts $[3,8,9,12]$. In adenocarcinoma arising in a tailgut cyst, elevation of a tumor marker such as CEA and CA19-9 is occasionally observed; in such cases, the tumor marker level can then be used to monitor their progression and recurrence [6, 12,13]. Complete surgical resection is the treatment of choice for eliminating the potential of recurrence, hemorrhage, infection, compression and malignant transformation. It also provides a specimen that is sufficient for histopathological examination to give a definite diagnosis $[6,14]$. Several methods of surgical approach are suggested for the resection of tailgut cyst. These include anterior (laparotomy or laparoscopic), posterior (perianal intersphincteric or parasacrococcygeal) or transanal en- doscopic microsurgery. An anterior approach via laparotomy is recommended when the size of a tumor is about $5 \mathrm{~cm}$ and the upper border of the tumor is located above the third sacrum and the lower border of the tumor does not reach the fourth sacrum $[11,15]$. The posterior approach is recommended for a low-lying tumor under the sacral promontory or below the level of the fourth sacrum. Transanal endoscopic microsurgery provides a good visual field for accurate excision and the laparoscopic approach has a advantage of a magnifying effect in the narrow pelvis without evidence of malignancy [16, 17]. In our case, the patient was diagnosed as double tailgut cysts without malignancy in pelvic MRI and PET scan. The larger tailgut cyst, measuring over $5 \mathrm{~cm}$ in size, was formed largely across the sacrum, hence, combined anterior-posterior approach was needed for complete resection of tumors, but both tumors was resected completely via anterior approach.

\section{CONCLUSION}

In conclusion, although tailgut cysts are rare and preoperative diagnosis is difficult, the possibility of these cysts should be considered in evaluating a patient complaining of anal symptoms, especially recurrent inflammatory processes of the perianal area. With detailed history taking and physical assessment, MRI is a helpful technique to define the extent of the tailgut cyst, its relationship to the surrounding structures and also to choose the best surgical approaches for complete resection.

\section{REFERENCES}

[1] Gips, M., Melki, Y. and Wolloch, Y. (1994) Cysts of the tailgut. Two cases. European Journal of Surgery, 160, 459-460.

[2] Jang, S.H., Jang, K.S., Song, Y.S., Min, K.W., Han, H.X., Lee, K.G. and Paik, S.S. (2006) Unusual prerectal location of a tailgut cyst: A case report. World Journal of Gastroenterology, 12, 5081-5083.

[3] Tampi, C., Lotwala, V., Lakdawala, M. and Coelho, K. (2007) Retrorectal cyst hamartoma (tailgut cyst) with malignant transformation. Gynecologic Oncology, 105, 266268. doi:10.1016/j.ygyno.2007.01.008

[4] Dahan, H., Arrivé, L., Wendum, D., Docou le Pointe, H., Djouhri, H. and Tubiana, J.M. (2001) Retrorectal developmental cysts in adults: Clinical and radiologi-histopathologic review, differential diagnosis, and treatment. $R a$ diographics, 21, 575-584.

[5] Hutton, K.A. and Benson, E.A. (1992) Case report: Tailgut cyst-assesment with transrectal ultrasound. Clinical Radiology, 45, 288-289. doi:10.1016/S0009-9260(05)80020-0

[6] Au, E., Anderson, O., Morgan, B., Alarcon, L. and George, M.L. (2009) Tailgut cysts: Report of two cases. International Journal of Colorectal Disease, 24, 345-350. 


\section{doi:10.1007/s00384-008-0598-6}

[7] Hjermstad, B.M. and Helwig, E.B. (1988) Tailgut cysts. Report of 53 cases. American Journal of Clinical Pathology, 89, 139-147.

[8] Gonul, I.I., Baglan, T., Pala, I. and Mentes, B. (2007) Tailgut cysts: Diagnostic challenge for both pathologists and clinicians. International Journal of Colorectal Diseases, 22, 1283-1285. doi:10.1007/s00384-006-0153-2

[9] Aflalo-Hazan, V., Rousset, P., Mourra, N., Lewin, M., Azizi, L. and Hoeffel, C. (2008) Tailgut cysts: MRI findings. European Radiology, 18, 2586-2593. doi:10.1007/s00330-008-1028-4

[10] Yang, D.M., Park, C.H., Jin, W., Chang, S.K., Kim, J.E., Choi, S.J. and Jung, D.H. (2005) Tailgut cyst: MRI evaluation. American Journal of Roentgenology, 184, 15191523. doi:10.2214/ajr.184.5.01841519

[11] Singer, M.A., Cintron, J.R., Martz, J.E., Schoetz, D.J. and Abcarian, H. (2003) Retrorectal cyst: A rare tumor frequently misdiagnosed. Journal of the American College of Surgeons, 196, 880-886. doi:10.1016/S1072-7515(03)00133-9

[12] Schwarz, R.E., Lyda, M., Lew, M. and Paz, I.B. (2000) A carcinoembryonic antigen-secreting adenocarcinoma arising within a retrorectal tailgut cyst: Clinicopathological considerations. American Journal of Gastroenterology, 95,
1344-1347. doi:10.1111/j.1572-0241.2000.02023.x

[13] Maruyama, A., Murabayashi, K., Hayashi, M., Nakano, H., Isaji, S., Uehara, S., Kusuda, T., Miyahara, S., Kondo, A., Nakano, H. and Yabana, T. (1998) Adenocarcinoma arising in a tailgut cyst: Report of a case. Surgery Today, 28, 1319-1322. doi:10.1007/BF02482826

[14] Sriganeshan, V. and Alexis, J.B. (2006) A 37-year-old woman with a presacral mass. Tailgut cyst (retrorectal cystic hamartoma). Archives of Pathology \& Laboratory Medicine, 130, 77-78.

[15] Glasgow, S.C., Birnbaum, E.H., Lowney, J.K., Fleshman, J.W., Kodner, I.J., Mutch, D.G., Lewin, S., Mutch, M.G. and Dietz, D.W. (2005) Retrorectal tumors: A diagnostic and therapeutic challenge. Diseases of the Colon \& Rectum, 48, 1581-1587. doi:10.1007/s10350-005-0048-2

[16] Buchs, N., Taylor, S. and Roche, B. (2007) The posterior approach for low retrorectal tumors in adults. International Journal of Colorectal Diseases, 22, 381-385. doi:10.1007/s00384-006-0183-9

[17] Zoller, S., Joos, A., Dinter, D., Back, W., Horisberger, K., Post, S. and Palma, P. (2007) Retrorectal tumors: Excision by transanal endoscopic microsurgery. Revista Espanola de Enfermedades Digestivas, 99, 547-550. doi:10.4321/S1130-01082007000900011 